Moroccan J. of Pure and Appl. Anal. (MJPAA)

Volume 7(3), 2021, Pages 413-429

ISSN: Online 2351-8227 - Print 2605-6364

DOI: $10.2478 / \mathrm{mjpaa}-2021-0028$

\title{
An efficient algorithm for solving the conformable time-space fractional telegraph equations
}

\author{
ABDELKEBIR SAAD ${ }^{1}$, NOUIRI BRAHIM ${ }^{2}$
}

AвSTRACT. In this paper, an efficient algorithm is proposed for solving one dimensional time-space-fractional telegraph equations. The fractional derivatives are described in the conformable sense. This algorithm is based on shifted Chebyshev polynomials of the fourth kind. The time-space fractional telegraph equations is reduced to a linear system of second order differential equations and the Newmark's method is applied to solve this system. Finally, some numerical examples are presented to confirm the reliability and effectiveness of this algorithm.

Mathematics Subject Classification (2020). 35R11; 26A33; 33C45.

Key words and phrases. Conformable fractional calculus, Newmark's method, Shifted Chebyshev polynomials of the fourth kind, Time-space-fractional telegraph equation.

\section{Introduction}

Telegraph equation is introduced by Oliver Heaviside and is a linear second-order hyperbolic partial differential equations, see [14]. This equation describe the current and voltage on an electrical transmission line with distance and demonstrates that the electromagnetic waves can

Received :August 31, 2020 - Accepted: April 08, 2021.

(C) The Author(s) 2021. This article is published with open access by Sidi Mohamed Ben Abdallah University.

${ }^{1}$ Department of Mathematics, University of M'sila, Algeria.

e-mail: saad.abdelkebir@univ-msila.dz

${ }^{2}$ Laboratory of Pure and Applied Mathematics, University of M'sila, Box 166, Ichbilia, 28000, M'sila, Algeria.

e-mail: brahim.nouiri@univ-msila.dz (Corresponding Author). 
be reflected on the wire, and that appear wave patterns along the transmission line [22]. The nonhomogeneous linear telegraph equation can be written as follows:

$$
\frac{\partial^{2} u(x, t)}{\partial t^{2}}+\left(\frac{R}{L}+\frac{G}{C}\right) \frac{\partial u(x, t)}{\partial t}+\frac{R G}{L C} u(x, t)=\frac{1}{L C} \frac{\partial^{2} u(x, t)}{\partial x^{2}}+f(x, t),
$$

where $u(x, t)$ can be voltage or current through the wire at position $x$ and time $t, R$ and $G$ are, respectively, the resistance and the conductance of resistor, $C$ is the capacitance of capacitor, and $L$ is the inductance of coil. This telegraph equation is applicable in several fields such as wave propagation [43], random walk theory [2], signal analysis [17].

Various numerical scheme such as splines radial basis function [13], Chebyshev Tau method [34], Legendre multiwavelet Galerkin method [45], homotopy perturbation method [24], Chebyshev spectral collocation method [16], differential quadrature method [30], B-spline collocation method [38], Haar wavelet method [3], Bessel functions [10] and dual reciprocity boundary integral equation method [12] have been applied to solve telegraph equation.

Fractional calculus, as an extension of the classical derivatives and integrals to non-integer orders, has been frequently used to model many fundamental problems in various branches of sciences and engineering [31,36, 8]. More recently, it has been found that fractional operators are more suitable for modeling phenomena in sciences and engineering.

In 2014, a new definition of fractional derivative, named "conformable fractional derivative", is introduced by Khalil et al. [18]. This novel fractional derivative is compatible with the classical derivative and it is excellent for studying non regular solutions. The subject of the conformable fractional derivative has attracted the attention of many authors in domains such as mechanics, electronic, and anomalous diffusion. We are interested in studying in this paper the telegraph model (1.1) in framework of the conformable time-space-fractional derivative. Precisely, we will propose the following transformations:

$$
\left\{\begin{array}{l}
\frac{\partial}{\partial t} \rightarrow \mathcal{D}_{t}^{(\alpha)}, \frac{\partial^{2}}{\partial t^{2}} \rightarrow \mathcal{D}_{t}^{(1+\alpha)} \text { and } \frac{\partial^{2}}{\partial x^{2}} \rightarrow \mathcal{D}_{x}^{(\beta)}, \\
2 a=R / L+G / C, \quad b^{2}=R G / L C, \quad \omega=1 / L C, \quad 0<\alpha \leq 1, \quad 1<\beta \leq 2
\end{array}\right.
$$

where $\mathcal{D}_{t}^{(\alpha)}$ and $\mathcal{D}_{x}^{(\beta)}$ are the conformable time and space fractional derivative operators [18]. Then, we get the conformable time-space-fractional telegraph model associated with the transformation (1.2) as follows:

$$
\mathcal{D}_{t}^{(1+\alpha)} u(x, t)+2 a \mathcal{D}_{t}^{(\alpha)} u(x, t)+b^{2} u(x, t)=\omega \mathcal{D}_{x}^{(\beta)} u(x, t)+f(x, t),
$$

subject to the initials conditions

$$
u(x, 0)=\varphi(x), \quad \mathcal{D}_{t}^{(\alpha)} u(x, 0)=\psi(x), \quad 0 \leq x \leq 1,
$$

and the Dirichlet boundary condition

$$
u(0, t)=g(t) \text { and } u(1, t)=h(t), \quad 0 \leq t \leq T,
$$

where $\varphi, \psi \in \mathcal{C}^{2}(0,1), g, h \in \mathcal{C}^{2}(0, T)$ and $f \in \mathcal{C}([0,1] \times[0, T])$ are given functions.

Numerical solution of fractional telegraph equation have been investigated by many authors. Mollahasani et. al considered the time-fractional telegraph equations and used hybrid Legendre functions to approximate their solutions [25]. In order to solve two-dimensional 
fractional telegraph equation a spectral meshless radial point interpolation method was proposed in [39]. Bhrawy et al. proposed a Chebyshev Tau method for numerical solution of the two-sided fractional-order telegraph equation [4]. A computational Tau method based on the Legendre polynomials has been proposed to solve time-fractional telegraph equations by Saadatmandi and Mohabbati [35]. Suleman et. al [41] have been used a new projected differential transform method for space and time fractional telegraph equations. In [9] the method of separation of variables has been applied for deriving the analytical solutions of time-fractional telegraph equations with different kind of boundary conditions. Sweilam et. al [42] considered the Sinc-Legendre collocation method for solving time-fractional telegraph equations. Fourier and Laplace transforms have been also applied to derive the analytical solutions of time-fractional telegraph equations $[19,20]$. Heydari et. al used an efficient Legendre wavelets method for numerical solution of time-fractional telegraph equations [15]. Moreover, semi-analytical methods have been employed by the researchers in [26, 44, 32, 37] for solving time-fractional telegraph equations.

In the last decade, considerable attention was paid to the application of orthogonal polynomials in the solution of fractional differential equations, integral equations and fractional partial differential equations. Numerical method based on Legendre [11, 35], Chebyshev [5, 34, 16], second kind of Chebyshev [33], fourth kind Chebyshev [6] and Jacobi [40, 27] polynomials were proposed. The rest of this paper is structured as follows: Section 2 deals with some description of conformable fractional derivative and its properties. In Section 3, deals with some properties of shifted Chebyshev polynomials of the fourth kind. Section 4 is devoted to evaluation of the conformable fractional derivative using shifted Chebyshev polynomials of the fourth kind. In Section 5, a Chebyshev collocation method based on the shifted Chebyshev polynomials of the fourth kind has been proposed to solve the problem (1.3)-(1.5). In Section 6, the Newmark's method has been proposed to solve this system. Various illustrative examples are considered to confirm accuracy of the Chebyshev collocation method in Section 7.

\section{Description of conformable fractional derivative and its properties}

Definition 2.1 ([18]). For a function $f:] 0,+\infty[\rightarrow \mathbb{R}$, the conformable fractional derivative of $f$ of order $0<\alpha<1$ in the variable $t$ is defined as:

$$
\mathcal{D}_{t}^{(\alpha)} f(t):=\lim _{\varepsilon \rightarrow 0} \frac{f\left(t+\varepsilon t^{1-\alpha}\right)-f(t)}{\varepsilon} .
$$

Some important properties of the conformable fractional derivative are as follows:

Theorem 2.1 ([18, 1]). (1) The conformable fractional derivative satisfies linearity properties similar to integer order differentiation

$$
\mathcal{D}_{x}^{(\alpha)}(\lambda f+\mu g)(x)=\lambda \mathcal{D}_{x}^{(\alpha)} f(x)+\mu \mathcal{D}_{x}^{(\alpha)} g(x), \quad \forall \lambda, \mu \in \mathbb{R} .
$$

(2) If $f$ is $n$ times differentiable, then we have

$$
\mathcal{D}_{t}^{(\alpha)} f(t)=t^{n+1-\alpha} f^{(n+1)}(t), \text { for all } n<\alpha \leq n+1 .
$$


(3) For conformable fractional derivative, we have

$$
\begin{aligned}
& \mathcal{D}^{(\alpha)} K=0, K \text { is a constant, } \\
& \mathcal{D}_{t}^{(\alpha)} t^{p}= \begin{cases}\frac{\Gamma(p+1)}{\Gamma(p-n)} t^{p-\alpha} & \text { if } p \in \mathbb{N} \text { and } p>\alpha, \\
0 & \text { if } p \in \mathbb{N} \text { and } p<\alpha,\end{cases}
\end{aligned}
$$

where $\Gamma$ denotes Gamma function and $n<\alpha \leq n+1$.

\section{Some properties of shifted Chebyshev polynomials of the fourth kind}

Shifted Chebyshev polynomials of the fourth kind $W_{n}^{*}(x)$ of degree $n$ in $x$ are defined on $[0,1]$ and can be determined the following recurrence formula, see [21].

$$
\left\{\begin{array}{l}
W_{0}^{*}(x)=1 \\
W_{1}^{*}(x)=4 x-1, \\
W_{n}^{*}(x)=2(2 x-1) W_{n-1}^{*}(x)-W_{n-2}^{*}(x), n=2,3, \ldots
\end{array}\right.
$$

The analytical form of the shifted Chebyshev polynomials of the fourth kind $W_{n}^{*}(x)$ of degree $n$ in $x$ is given by

$$
W_{n}^{*}(x)=\sum_{k=0}^{n}(-1)^{k} 2^{2 n-2 k} \frac{\Gamma(2 n-k+1)}{\Gamma(k+1) \Gamma(2 n-2 k+1)} x^{n-k}, n \in \mathbb{N}^{*} .
$$

These polynomials are orthogonal on the support interval $[0,1]$ as the following inner product:

$$
\left\langle W_{n}^{*}, W_{m}^{*}\right\rangle:=\int_{0}^{1} \sqrt{\frac{1-x}{x}} W_{n}^{*}(x) W_{m}^{*} d x= \begin{cases}0 & \text { if } n \neq m, \\ \pi / 2 & \text { if } n=m,\end{cases}
$$

where $x \mapsto \sqrt{\frac{1-x}{x}}$ is weight function corresponding to $W_{n}^{*}$.

Any function $y(x)$ belongs to the space of square integrable functions in $[0,1]$, may be expressed in terms of shifted Chebyshev polynomials of the fourth kind as

$$
y(x)=\sum_{i=0}^{+\infty} c_{i} W_{i}^{*}(x),
$$

where the coefficients $c_{i}, i \in \mathbb{N}$ are given by

$$
c_{i}=\frac{2}{\pi} \int_{0}^{1} y(x) \sqrt{\frac{1-x}{x}} W_{i}^{*}(x) d x .
$$

For practical purpose we take only first $(m+1)$-terms of $W_{n}^{*}(x)$ in approximation which is given:

$$
y_{m}(x)=\sum_{i=0}^{m} c_{i} W_{i}^{*}(x)
$$




\section{Evaluation of the conformable fractional derivative using shifted Chebyshev polynomi- als of the fourth kind}

The main approximate formula for the function $y_{m}(x)$ given in (3.3) is presented in the following theorem.

Theorem 4.1. Let $y_{m}(x)$ be approximated function in terms of shifted Chebyshev polynomials of the fourth kind as given in (3.3), suppose $\gamma>0$, then we have

$$
\mathcal{D}^{(\gamma)} y_{m}(x)=\sum_{i=n+1}^{m} \sum_{k=0}^{i-n-1} c_{i} \mathcal{N}_{i, k}^{n} x^{i-k-\gamma}, n<\gamma \leq n+1,
$$

where $\mathcal{N}_{i, k}^{n}$ is given by

$$
\mathcal{N}_{i, k}^{n}=(-1)^{k} 2^{2 i-2 k} \frac{\Gamma(2 i-k+1) \Gamma(i-k+1)}{\Gamma(k+1) \Gamma(2 i-2 k+1) \Gamma(i-k-n)} .
$$

Proof. By using the linearity of the conformable fractional derivative given in (2.1) and by using definition of approximation function $y_{m}(x)$ as in (3.3), we obtain

$$
\mathcal{D}_{x}^{(\gamma)} y_{m}(x)=\sum_{i=0}^{m} c_{i} \mathcal{D}_{x}^{(\gamma)} W_{i}^{*}(x), \text { for all } \gamma>0 .
$$

Moreover, from (2.1), (2.2), (2.4) and (3.1), we get

$$
\mathcal{D}_{x}^{(\gamma)} W_{i}^{*}(x)=0, \quad i=0,1, \ldots, n, n \in \mathbb{N}, n<\gamma \leq n+1 .
$$

For $i-k>n$ and from (2.4), we have

$$
\mathcal{D}_{x}^{(\gamma)} x^{i-k}=\frac{\Gamma(i-k+1)}{\Gamma(i-k-n)} x^{i-k-\gamma}, n \in \mathbb{N}, n<\gamma \leq n+1 .
$$

Substituting (4.5) into (3.1), we obtain

$$
\mathcal{D}_{x}^{(\gamma)} W_{i}^{*}(x)=\sum_{k=0}^{i-n-1}(-1)^{k} 2^{2 i-2 k} \frac{\Gamma(2 i-k+1) \Gamma(i-k+1)}{\Gamma(k+1) \Gamma(2 i-2 k+1) \Gamma(i-k-n)} x^{i-k-\gamma} .
$$

Substituting (4.6) into (4.3), we obtain for $n<\gamma \leq n+1$ :

$$
\mathcal{D}_{x}^{(\gamma)} y_{m}(x)=\sum_{i=n+1}^{m} \sum_{k=0}^{i-n-1} c_{i}(-1)^{k} 2^{2 i-2 k} \frac{\Gamma(2 i-k+1) \Gamma(i-k+1)}{\Gamma(k+1) \Gamma(2 i-2 k+1) \Gamma(i-k-n)} x^{i-k-\gamma},
$$

where can be rewritten as the form

$$
\mathcal{D}_{x}^{(\gamma)} y_{m}(x)=\sum_{i=n+1}^{m} \sum_{k=0}^{i-n-1} c_{i} \mathcal{N}_{i, k}^{n} x^{i-k-\gamma},
$$

where $\mathcal{N}_{i, k}^{n}$ is defined by (4.2). 
Example 4.1. Consider $g(x)=x^{2}$ with $m=2, \gamma=1.8$ and $n=1$. Using (2.3) and (2.4), we obtain

$$
\mathcal{D}_{x}^{(1.8)} x^{2}=\mathcal{D}_{x}^{(0.8)}(2 x)=2 \mathcal{D}_{x}^{(0.8)}(x)=2 x^{0.2} .
$$

Now, using Theorem 4.1 we obtain:

$$
\mathcal{D}_{x}^{(1.8)} x^{2}=c_{2} \mathcal{N}_{2,0}^{1} x^{0.2}
$$

From (3.2) and (4.2), we obtain:

$$
c_{2}=\frac{1}{16} \text { and } \mathcal{N}_{2,0}^{1}=32
$$

Then, $\mathcal{D}_{x}^{(1.8)} x^{2}=2 x^{0.2}$.

4.1. Error Analysis. The main goal of this section is study the truncating error and its convergence analysis.

Theorem 4.2. (Uniformly converges theorem, [6]). Let $y \in L^{2}(0,1)$ be a function twice differentiable on $[0,1]$ and the second derivative is bounded on $[0,1]$ i.e.

$$
\exists M>0, \forall x \in[0,1]:\left|y^{\prime \prime}(x)\right| \leq M \text {. }
$$

If $y(x)=\sum_{i=0}^{+\infty} c_{i} W_{i}^{*}(x)$ is the sum of the series of shifted Chebyshev polynomials of the fourth kind, then the sequence of partial sums $\left(y_{m}\right)$ with $y_{m}(x)=\sum_{i=0}^{m} c_{i} W_{i}^{*}(x)$ uniformly converges to $y(x)$ on $[0,1]$.

Proof. Using the variable change $2 x-1=\cos (\theta)$ in (3.2), we obtain:

$$
c_{i}=\frac{2}{\pi} \int_{0}^{\pi} y\left(\frac{1+\cos (\theta)}{2}\right) \sin \left[\left(i+\frac{1}{2}\right) \theta\right] \sin (\theta / 2) d \theta .
$$

Now integration by parts two times, we get:

$$
c_{i}=\frac{1}{4 \pi} \int_{0}^{\pi} y^{\prime \prime}\left(\frac{1+\cos (\theta)}{2}\right) \delta_{i}(\theta) d \theta
$$

where

$$
\begin{gathered}
\delta_{i}(\theta)=\sin (\theta)\left[\frac{1}{i}\left(\frac{\sin (i-1) \theta}{i-1}-\frac{\sin (i+1) \theta}{i+1}\right)+\frac{1}{i+1}\left(\frac{\sin i \theta}{i}-\frac{\sin (i+2) \theta}{i+2}\right)\right] . \\
\left|c_{i}\right|=\left|\frac{1}{4 \pi} \int_{0}^{\pi} y^{\prime \prime}\left(\frac{1+\cos (\theta)}{2}\right) \delta_{i}(\theta) d \theta\right| \leq \frac{M}{4 \pi} \int_{0}^{\pi}\left|\delta_{i}(\theta)\right| d \theta=\frac{M\left(i^{2}+2 i-1\right)}{i\left(i^{2}-1\right)(i+2)} \leq \frac{M}{i^{2}} .
\end{gathered}
$$

Other hand, we have:

$$
\left|y(x)-y_{m}(x)\right| \leq \sum_{i=m+1}^{+\infty}\left|c_{i}\right|\left|W_{i}^{*}(x)\right| \leq \sum_{i=m+1}^{+\infty}\left|c_{i}\right| \leq \sum_{i=m+1}^{+\infty} \frac{M}{i^{2}}
$$


Then, $\sum_{i=1}^{+\infty} \frac{1}{i^{2}}$ is the Riemann series converges, then the rest of this series converges to zero, so the sequence $\left(y_{m}(x)\right)$ is uniformly converges to $y(x)$ on $[0,1]$.

\section{Chebyshev Collocation Method}

In this section, we apply Chebyshev collocation method to problems (1.3)-(1.5) based on the shifted Chebyshev polynomials of the fourth kind. Let us denote $u_{m}(x, t)$ as the approximation to $u(x, t)$ in the following form

$$
u_{m}(x, t)=\sum_{i=0}^{m} c_{i}(t) W_{i}^{*}(x)
$$

We apply Theorem 4.1, (1.3) and (5.1), we obtain

$$
\begin{aligned}
\sum_{i=0}^{m} t^{1-\alpha} c_{i}^{\prime \prime}(t) W_{i}^{*}(x) & +2 a \sum_{i=0}^{m} t^{1-\alpha} c_{i}^{\prime}(t) W_{i}^{*}(x)+b^{2} \sum_{i=0}^{m} c_{i}(t) W_{i}^{*}(x) \\
& =\omega \sum_{i=2}^{m} \sum_{k=0}^{i-2} c_{i}(t) \mathcal{N}_{i, k}^{1} x^{i-k-\beta}+f(x, t)
\end{aligned}
$$

In order to find the unknown coefficients, Chebyshev collocation method with the following collocation points

$$
x_{p}=\frac{1}{2}(1+\cos (p \pi / m)), p=1,2, \ldots, m-1,
$$

are applied. Then, we have

$$
\sum_{i=0}^{m}\left[t^{1-\alpha} c_{i}^{\prime \prime}(t) W_{i}^{*}\left(x_{p}\right)+2 a t^{1-\alpha} c_{i}^{\prime}(t) W_{i}^{*}\left(x_{p}\right)+c_{i}(t) R_{i}\left(x_{p}\right)\right]=f\left(x_{p}, t\right),
$$

where

$$
\left\{\begin{array}{l}
S_{0}\left(x_{p}\right)=S_{1}\left(x_{p}\right)=0, \\
S_{i}\left(x_{p}\right)=\sum_{k=0}^{i-2} \mathcal{N}_{i, k}^{1} x_{p}^{i-k-\beta}, i=2,3, \ldots, m, \\
R_{i}\left(x_{p}\right)=b^{2} W_{i}^{*}\left(x_{p}\right)-\omega S_{i}\left(x_{p}\right), i=1,2, \ldots, m
\end{array}\right.
$$

Also put (5.1) in (1.5), we get

$$
\sum_{i=0}^{m}(-1)^{i} c_{i}(t)=g(t), \sum_{i=0}^{m}(2 i+1) c_{i}(t)=h(t) .
$$

We introduce the vectors $V(t)$ and $F(t)$ defined by

$$
\begin{aligned}
& V(t)=\left(c_{0}(t), c_{1}(t), \ldots, c_{m}(t)\right)^{T} \\
& F(t)=\left(f\left(x_{1}, t\right), f\left(x_{2}, t\right), \ldots, f\left(x_{m-1}, t\right), g(t), h(t)\right)^{T} .
\end{aligned}
$$


Substituting (3.2) and (5.1) into the initial conditions (1.4), we can compute

$$
V(0)=\left(c_{0}(0), c_{1}(0), \ldots, c_{m}(0)\right)^{T} \text { and } \frac{d}{d t} V(0)=\left(c_{0}^{\prime}(0), c_{1}^{\prime}(0), \ldots, c_{m}^{\prime}(0)\right)^{T} .
$$

Let the matrices $M(t), C(t)$ and $K$ given by

$$
\begin{aligned}
& M(t)=t^{1-\alpha}\left(\begin{array}{cccc}
W_{0}^{*}\left(x_{1}\right) & W_{1}^{*}\left(x_{1}\right) & \ldots & W_{m}^{*}\left(x_{1}\right) \\
W_{0}^{*}\left(x_{2}\right) & W_{1}^{*}\left(x_{2}\right) & \ldots & W_{m}^{*}\left(x_{2}\right) \\
\vdots & \ddots & \ldots & \vdots \\
W_{0}^{*}\left(x_{m-1}\right) & W_{1}^{*}\left(x_{m-1}\right) & \ldots & W_{m}^{*}\left(x_{m-1}\right) \\
0 & 0 & \ldots & 0 \\
0 & 0 & \ldots & 0
\end{array}\right), \\
& C(t)=2 a M, \\
& K=\left(\begin{array}{cccc}
R_{0}\left(x_{1}\right) & R_{1}\left(x_{1}\right) & \ldots & R_{m}\left(x_{1}\right) \\
R_{0}\left(x_{2}\right) & R_{1}\left(x_{2}\right) & \ldots & R_{m}\left(x_{2}\right) \\
\vdots & \ldots & \ddots & \vdots \\
R_{0}\left(x_{m-1}\right) & R_{1}\left(x_{m-1}\right) & \ldots & R_{m}\left(x_{m-1}\right) \\
1 & -1 & \ldots & (-1)^{m} \\
1 & 3 & \ldots & 2 m+1
\end{array}\right)
\end{aligned}
$$

By combining the equations (5.3) and (5.4), we find the following matrix form

$$
\left\{\begin{array}{l}
M(t) \ddot{V}(t)+C(t) \dot{V}(t)+K V(t)=F(t), \\
V(0)=\left(c_{0}(0), c_{1}(0), \ldots, c_{m}(0)\right)^{T} \\
\dot{V}(0)=\left(c_{0}^{\prime}(0), c_{1}^{\prime}(0), \ldots, c_{m}^{\prime}(0)\right)^{T} .
\end{array}\right.
$$

To solve the system of second order differential equations (5.5), we use Newmark's method.

\section{Newmark's method}

For positive integer $N, \Delta t=T / N$, denotes the step size of the variable $t$. So we define $t_{j}=$ $j \Delta t$ in which $j=0,1, \ldots, N$, and we introduce the following notations: $c_{i}\left(t_{j}\right)=c_{i}^{j}, g\left(t_{j}\right)=g^{j}$, $h\left(t_{j}\right)=h^{j}, V^{j}=\left(c_{0}^{j}, c_{1}^{j}, \ldots, c_{m}^{j}\right)^{T}$ and $F^{j}=\left(f\left(x_{1}, t_{j}\right), f\left(x_{2}, t_{j}\right), \ldots, f\left(x_{m-1}, t_{j}\right), g^{j}, h^{j}\right)^{T}$.

In 1959 Newmark proposed a method, see [29] which links the accelerations, the velocities and the displacements of the nodes at the instants $t_{j+1}$ and $t_{j}$ as follows:

$$
\begin{aligned}
& V^{j+1}=V^{j}+\Delta t \dot{V}^{j}+\frac{(\Delta t)^{2}}{2}\left[(1-2 \theta) \ddot{V}^{j}+2 \theta \ddot{V}^{j+1}\right], \\
& \dot{V}^{j+1}=\dot{V}^{j}+\Delta t\left[(1-\gamma) \ddot{V}^{j}+\gamma \ddot{V}^{j+1}\right] .
\end{aligned}
$$

where $0 \leq \gamma \leq 1$ and $0 \leq \theta \leq 1 / 2$. The most commonly used method is the average acceleration method $(\theta=1 / 4$ and $\gamma=1 / 2)$, see [7]. 
We then try to solve the system (5.5) at the instant $t_{j+1}$ :

$$
M^{j+1} \ddot{V}^{j+1}+C^{j+1} \dot{V}^{j+1}+K V^{j+1}=F^{j+1}, j=0,1, \ldots, N-1 .
$$

Rearranging (6.1) to provide an expression for $\ddot{V}^{j+1}$ gives:

$$
\ddot{V}^{j+1}=\frac{V^{j+1}-V^{j}}{\theta \Delta t^{2}}-\frac{\dot{V}^{j}}{\theta \Delta t}-\left(\frac{1}{2 \theta}-1\right) \ddot{V}^{j} .
$$

Inserting the expression for $\ddot{V}^{j+1}$ from (6.4) into (6.2) gives:

$$
\dot{V}^{j+1}=\frac{\gamma}{\theta \Delta t}\left(V^{j+1}-V^{j}\right)+\left(1-\frac{\gamma}{\theta}\right) \dot{V}^{j}+\Delta t\left(1-\frac{\gamma}{2 \theta}\right) \ddot{V}^{j}
$$

We can now insert the explicit expressions for $\ddot{V}^{j+1}$ and $\dot{V}^{j+1}$ from (6.4) and (6.5) into (6.3) to obtain the following linear system:

$$
A^{j+1} V^{j+1}=B^{j+1}
$$

where

$$
\begin{aligned}
A^{j+1} & =\frac{M^{j+1}}{\theta(\Delta t)^{2}}+\frac{\gamma C^{j+1}}{\theta \Delta t}+K, \\
B^{j+1} & =F^{j+1}+M^{j+1}\left[\frac{V^{j}}{\theta(\Delta t)^{2}}+\frac{\dot{V}^{j}}{\theta \Delta t}+\left(\frac{1}{2 \theta}-1\right) \ddot{V}^{j}\right] \\
& +C^{j+1}\left[\frac{\gamma V^{j}}{\theta \Delta t}-\left(1-\frac{\gamma}{\theta}\right) \dot{V}^{j}-\Delta t\left(1-\frac{\gamma}{2 \theta}\right) \ddot{V}^{j}\right] .
\end{aligned}
$$

The complete algorithm using the Newmark's method is given in the following: 
Algorithm 1 (Newmark's method)

1: Initial calculations:

(1) Form stiffness matrix $K$, mass matrix $M^{j+1}$, and damping matrix $C^{j+1}$.

(2) Initialize $V^{0}, \dot{V}^{0}$, and $\ddot{V}^{0}$.

(3) Select time step $\Delta t=T / N$, parameter $\theta, \gamma$ and calculate integration constants:

$$
\begin{aligned}
& \gamma \geq 1 / 2 ; \theta \geq \frac{(\gamma+1 / 2)^{2}}{4} ; \\
& a_{0}=\frac{1}{\theta(\Delta t)^{2}} ; a_{1}=\frac{\gamma}{\theta \Delta t} ; a_{2}=\frac{1}{\theta \Delta t} ; a_{3}=\frac{1}{2 \theta}-1 ; \\
& a_{4}=\frac{\gamma}{\theta}-1 ; a_{5}=\Delta t\left(\frac{\gamma}{2 \theta}-1\right) ; a_{6}=\Delta t(1-\gamma) ; a_{7}=\gamma \Delta t .
\end{aligned}
$$

(4) Form effective stiffness matrix $A^{j+1}=a_{0} M^{j+1}+a_{1} C^{j+1}+K$.

\section{2: For each time step:}

(1) Calculate effective loads at time $t_{j+1}=t_{j}+\Delta t$ :

$$
B^{j+1}=F^{j+1}+M^{j+1}\left(a_{0} V^{j}+a_{2} \dot{V}^{j}+a_{3} \ddot{V}^{j}\right)+C^{j+1}\left(a_{1} V^{j}+a_{4} \dot{V}^{j}+a_{5} \ddot{V}^{j}\right) .
$$

(2) Solve for displacements at time $t_{j+1}=t_{j}+\Delta t$ :

$$
A^{j+1} V^{j+1}=B^{j+1} \text {. }
$$

(3) Calculate accelerations and velocities at time $t_{j+1}=t_{j}+\Delta t$ :

$$
\begin{aligned}
& \ddot{V}^{j+1}=a_{0}\left(V^{j+1}-V^{j}\right)-a_{2} \dot{V}^{j}-a_{3} \ddot{V}^{j}, \\
& \dot{V}^{j+1}=\dot{V}^{j}+a_{6} \ddot{V}^{j}+a_{7} \ddot{V}^{j+1} .
\end{aligned}
$$

\section{Applications}

In this section, we present some numerical results of the conformable time-space fractional telegraph equations (1.3) with initial (1.4) and boundary conditions (1.5). To test the efficiency of Algorithm 1 several numerical examples for different values of $a, b, \omega, \alpha$ and $\beta$ are given in this section with $L_{2}, L_{\infty}$ and root mean square (RMS) errors are calculated by

$$
\begin{aligned}
L_{2} & =\left\|u_{e}-u_{m}\right\|_{2}=\sqrt{h \sum_{i=0}^{N}\left|u_{e}\left(x_{i}, t\right)-u_{m}\left(x_{i}, t\right)\right|^{2},} \\
L_{\infty} & =\left\|u_{e}-u_{m}\right\|_{\infty}=\max _{0 \leq i \leq N}\left|u_{e}\left(x_{i}, t\right)-u_{m}\left(x_{i}, t\right)\right| \\
R M S & =\sqrt{\frac{\sum_{i=0}^{N}\left|u_{e}\left(x_{i}, t\right)-u_{m}\left(x_{i}, t\right)\right|^{2}}{N+1}},
\end{aligned}
$$

where $u_{e}$ is the exact solution and $u_{m}$ is the numerical solution. 
We compare the numerical solutions obtained by Algorithm 1 with known exact solutions and those numerical methods available in the literature. All numerical computations were carried out by MATLAB R2014b in OS Windows 10 (64 bits) with Intel(R) Core(TM) i7-2670QM, 2.20 GHz CPU machine and 8 GB of memory.

Example 7.1. In this example, we consider the telegraph Eq.(1.3) with $a=1 / 2, b=1, \omega=1$ and $\beta=2$ in the domain $0 \leq x \leq 1$, with following initial and boundary conditions,

$$
\left\{\begin{array}{l}
u(x, 0)=\mathcal{D}_{t}^{(\alpha)} u(x, 0)=0,0 \leq x \leq 1, \\
u(0, t)=u(1, t)=0,0<t \leq 1 .
\end{array}\right.
$$

and $f(x, t)=\left(\alpha+\alpha^{2}-(1+\alpha) t+t^{1+\alpha}\right)\left(x-x^{2}\right) e^{-t}+2 t^{1+\alpha} e^{-t}$. The exact solution of this example is $u_{e}(x, t)=\left(x-x^{2}\right) t^{1+\alpha} e^{-t}$. If $\alpha=1$, this example was studied in $[23,28]$ by different numerical methods. We apply Algorithm 1 for $m=5$ and approximate to the solution $u(x, t)$ as follows:

$$
u_{5}(x, t)=\sum_{i=0}^{5} c_{i}(t) W_{i}^{*}(x) .
$$

Using the linear system (6.6) for $m=5$ and $N=1000$ with the initial data $V^{0}=\dot{V}^{0}=\ddot{V}^{0}=$ $(0,0,0,0,0,0)^{T}$. The $L_{2}$ and $L_{\infty}$ errors and CPU time in seconds is shown in Table 1 . Numerical results are compared with the obtained results in [23] and [28]. It can be concluded that the numerical solutions obtained by our algorithm are good. In Table 2, we present $L_{2}$ and $L_{\infty}$ errors at different $t$ for $\alpha=0.2,0.4,0.6,0.8,1.0$, with $\Delta t=0.0001$ and $h=0.01$. The graph of exact and numerical solutions for $\alpha=1$ at $t=1,2,3,4,5$ and for $t=2$ at $\alpha=0.2,0.4,0.6,0.8,1$ is shown in Figure 1 and the time-space graphs of numerical solution and absolute error up to $t=5$ are presented in Figure 2.

TABLE 1. $L_{2}$ and $L_{\infty}$ errors and CPU time of Example 7.1 with $\Delta t=0.001, h=0.01$ and $\alpha=1$

\begin{tabular}{|c|c|c|c|c|c|c|c|c|c|}
\cline { 2 - 10 } \multicolumn{1}{c|}{} & \multicolumn{3}{c|}{ Proposed Algorithm } & \multicolumn{3}{c|}{ CuTBSM, [28] } & \multicolumn{3}{c|}{ CuBSM, [23] } \\
\hline $\mathrm{T}$ & $L_{2}$ & $L_{\infty}$ & $\mathrm{CPU}(\mathrm{s})$ & $L_{2}$ & $L_{\infty}$ & $\mathrm{CPU}(\mathrm{s})$ & $L_{2}$ & $L_{\infty}$ & $\mathrm{CPU}(\mathrm{s})$ \\
\hline 1.0 & $8.15 \mathrm{E}-07$ & $1.46 \mathrm{E}-06$ & 0.24 & $6.31 \mathrm{E}-05$ & $8.76 \mathrm{E}-05$ & 0.34 & $4.55 \mathrm{E}-05$ & $5.91 \mathrm{E}-05$ & 0.43 \\
2.0 & $1.30 \mathrm{E}-05$ & $1.83 \mathrm{E}-05$ & 0.45 & $2.34 \mathrm{E}-05$ & $3.29 \mathrm{E}-05$ & 0.57 & $1.43 \mathrm{E}-05$ & $1.78 \mathrm{E}-05$ & 0.77 \\
3.0 & $4.75 \mathrm{E}-06$ & $7.12 \mathrm{E}-06$ & 0.65 & $4.62 \mathrm{E}-06$ & $5.90 \mathrm{E}-06$ & 1.05 & $6.42 \mathrm{E}-06$ & $1.43 \mathrm{E}-05$ & 1.15 \\
4.0 & $9.09 \mathrm{E}-06$ & $1.26 \mathrm{E}-05$ & 0.86 & $2.19 \mathrm{E}-05$ & $3.04 \mathrm{E}-05$ & 1.11 & $8.92 \mathrm{E}-06$ & $1.35 \mathrm{E}-05$ & 1.29 \\
5.0 & $4.43 \mathrm{E}-06$ & $6.03 \mathrm{E}-06$ & 1.07 & $5.18 \mathrm{E}-06$ & $6.92 \mathrm{E}-06$ & 1.26 & $3.01 \mathrm{E}-06$ & $5.20 \mathrm{E}-06$ & 1.46 \\
\hline
\end{tabular}

TABLE 2. $L_{2}$ and $L_{\infty}$ errors of Example 7.1 at different values of $T$ and $\alpha$ with $\Delta t=0.0001$ and $h=0.01$.

\begin{tabular}{|c|c|c|c|c|c|c|c|c|}
\cline { 2 - 9 } \multicolumn{1}{c|}{} & \multicolumn{2}{c|}{$T=1$} & \multicolumn{2}{c|}{$T=2$} & \multicolumn{2}{c|}{$T=3$} & \multicolumn{2}{c|}{$T=4$} \\
\hline$\alpha$ & $L_{2}$ & $L_{\infty}$ & $L_{2}$ & $L_{\infty}$ & $L_{2}$ & $L_{\infty}$ & $L_{2}$ & $L_{\infty}$ \\
\hline 0.2 & $2.51 \mathrm{E}-03$ & $3.53 \mathrm{E}-03$ & $2.08 \mathrm{E}-03$ & $2.93 \mathrm{E}-03$ & $7.99 \mathrm{E}-04$ & $1.12 \mathrm{E}-03$ & $6.14 \mathrm{E}-04$ & $8.58 \mathrm{E}-04$ \\
0.4 & $5.27 \mathrm{E}-04$ & $7.44 \mathrm{E}-04$ & $4.10 \mathrm{E}-04$ & $5.77 \mathrm{E}-04$ & $1.06 \mathrm{E}-04$ & $1.51 \mathrm{E}-04$ & $1.63 \mathrm{E}-04$ & $2.27 \mathrm{E}-04$ \\
0.6 & $7.65 \mathrm{E}-05$ & $1.09 \mathrm{E}-04$ & $6.24 \mathrm{E}-05$ & $8.91 \mathrm{E}-05$ & $1.51 \mathrm{E}-05$ & $2.21 \mathrm{E}-05$ & $2.82 \mathrm{E}-05$ & $3.91 \mathrm{E}-05$ \\
0.8 & $8.11 \mathrm{E}-06$ & $1.14 \mathrm{E}-05$ & $6.73 \mathrm{E}-06$ & $9.72 \mathrm{E}-06$ & $3.05 \mathrm{E}-06$ & $4.50 \mathrm{E}-06$ & $1.69 \mathrm{E}-06$ & $2.26 \mathrm{E}-06$ \\
1.0 & $6.95 \mathrm{E}-08$ & $1.26 \mathrm{E}-07$ & $1.25 \mathrm{E}-06$ & $1.75 \mathrm{E}-06$ & $5.64 \mathrm{E}-07$ & $8.38 \mathrm{E}-07$ & $1.03 \mathrm{E}-06$ & $1.43 \mathrm{E}-06$ \\
\hline
\end{tabular}




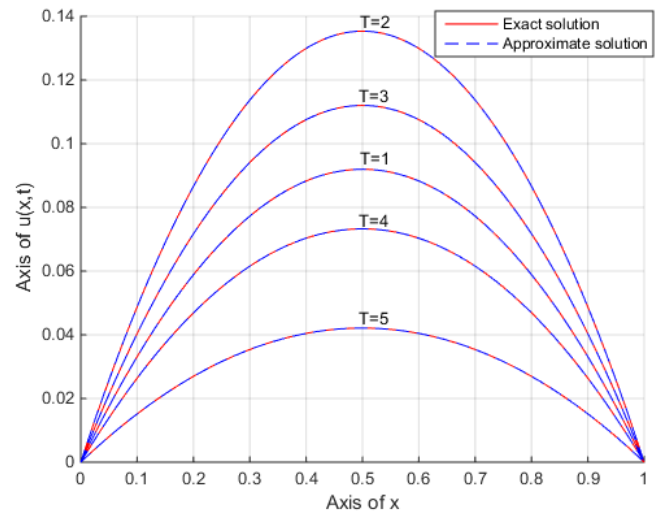

Figure (A). Exact and numerical solutions (Left) for $\alpha=1$ at $t=$ $1,2,3,4,5$.

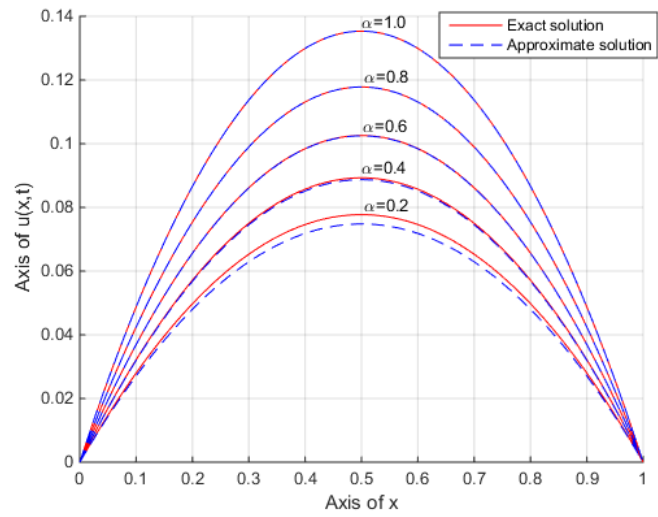

Figure (B). Exact and numerical solutions (Right) for $t=2$ at $\alpha=$ $0.2,0.4,0.6,0.8,1$.

FiguRE 1. Comparison of numerical and exact solutions of Example 7.1 at different time and $\alpha$ levels with $h=0.01, \Delta t=0.001$.

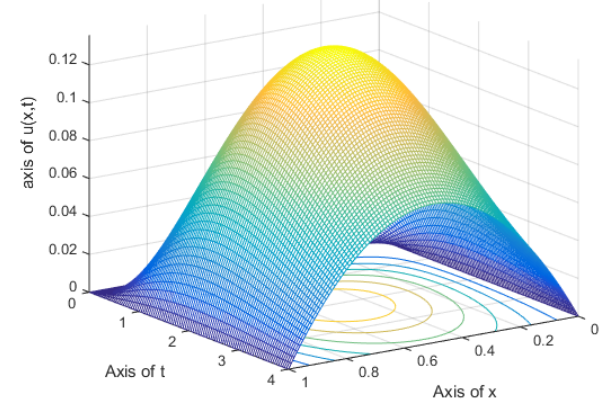

Figure (A). Exact solution.

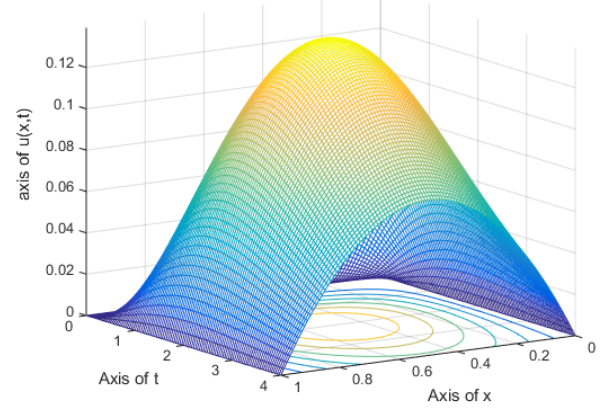

Figure (B). Numerical solution.

FIGURE 2. Time-space graphs of Exact and numerical solutions for Example 7.1 at $t=4$ and $\alpha=1$.

Example 7.2. We consider Eq.(1.3) in the domain $[0,1]$ with $a=6, b=2, \omega=1$ and $\alpha=1$. The initial and boundary conditions are given by,

$$
\begin{aligned}
& u(x, 0)=\sin ((\beta-1) x), u_{t}(x, 0)=0,0 \leq x \leq 1, \\
& u(0, t)=0, u(1, t)=\cos (t) \sin (\beta-1), t \geq 0
\end{aligned}
$$

and $f(x, t)=\left[3 \cos (t)-12 \sin (t)+(\beta-1)^{2} x^{2-\beta} \cos (t)\right] \sin (\beta-1) x$. The exact solution of this example is $u_{e}=\cos (t) \sin ((\beta-1) x)$. If $\beta=2$, this example was studied in [23, 28] by different 
numerical methods. We apply Algorithm 1 for $m=5$ and approximate to the solution $u(x, t)$ as follows:

$$
u_{5}(x, t)=\sum_{i=0}^{5} c_{i}(t) W_{i}^{*}(x) .
$$

Using the linear system (6.6) for $m=5$ and $N=1000$ with the initial data

$$
V^{0}=(0.2373,0.2273,-0.0124,-0.0023,0.0001,0)^{T}, \dot{V}^{0}=\ddot{V}^{0}=(0,0,0,0,0,0)^{T},
$$

we compute the approximate solution $u_{5}(x, t)$.

The efficiency of Algorithm 1 is measured using $L_{2}, L_{\infty}$ and root mean square errors with $\Delta t=$ $0.0001, h=0.01$ which are shown in Table 3.The numerical results are compared with the obtained results in $[28,23]$. It can be concluded that the numerical solutions obtained by our algorithm are good. In Figure 3, we present the comparison of numerical and exact solutions for different time levels with $\Delta t=0.001$ and $h=0.01$. Time-space graph of numerical solution up to $t=1$ and $t=2$ are shown in Figure 4.

TABLE 3. $L_{2}, L_{\infty}$ and RMS errors of Example 7.2 with $\Delta t=0.0001$ and $h=0.01$ and $\beta=2$.

\begin{tabular}{|c|c|c|c|c|c|c|c|c|c|}
\cline { 2 - 10 } \multicolumn{1}{c|}{} & \multicolumn{3}{c|}{ Proposed Algorithm } & \multicolumn{3}{c|}{ CuTBSM, [28] } & \multicolumn{3}{c|}{ CuBSM, [23] } \\
\hline $\mathrm{T}$ & $L_{2}$ & $L_{\infty}$ & RMS & $L_{2}$ & $L_{\infty}$ & RMS & $L_{2}$ & $L_{\infty}$ & RMS \\
\hline 0.2 & $5.07 \mathrm{E}-07$ & $1.03 \mathrm{E}-06$ & $5.09 \mathrm{E}-07$ & $2.96 \mathrm{E}-06$ & $4.63 \mathrm{E}-06$ & $2.94 \mathrm{E}-06$ & $2.69 \mathrm{E}-06$ & $5.24 \mathrm{E}-06$ & $2.67 \mathrm{E}-06$ \\
0.4 & $3.48 \mathrm{E}-07$ & $6.12 \mathrm{E}-07$ & $3.50 \mathrm{E}-07$ & $6.77 \mathrm{E}-06$ & $1.01 \mathrm{E}-05$ & $6.73 \mathrm{E}-06$ & $5.61 \mathrm{E}-06$ & $8.61 \mathrm{E}-06$ & $5.59 \mathrm{E}-06$ \\
0.6 & $9.23 \mathrm{E}-07$ & $1.70 \mathrm{E}-06$ & $9.28 \mathrm{E}-07$ & $9.81 \mathrm{E}-06$ & $1.42 \mathrm{E}-05$ & $9.76 \mathrm{E}-06$ & $9.75 \mathrm{E}-06$ & $1.25 \mathrm{E}-05$ & $9.70 \mathrm{E}-06$ \\
0.8 & $1.69 \mathrm{E}-06$ & $2.69 \mathrm{E}-06$ & $1.70 \mathrm{E}-06$ & $1.20 \mathrm{E}-05$ & $1.71 \mathrm{E}-05$ & $1.19 \mathrm{E}-05$ & $1.38 \mathrm{E}-05$ & $2.03 \mathrm{E}-05$ & $1.37 \mathrm{E}-05$ \\
1.0 & $2.01 \mathrm{E}-06$ & $2.91 \mathrm{E}-06$ & $2.02 \mathrm{E}-06$ & $1.34 \mathrm{E}-05$ & $1.90 \mathrm{E}-05$ & $1.33 \mathrm{E}-05$ & $1.73 \mathrm{E}-05$ & $2.75 \mathrm{E}-05$ & $1.72 \mathrm{E}-06$ \\
\hline
\end{tabular}

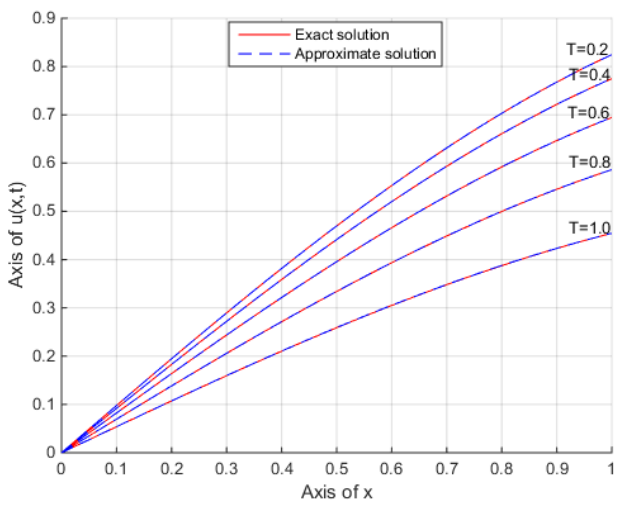

Figure (A). Exact and numerical solutions (Left) for $\beta=2$ at $T=0.2$ to $T=1.0$.

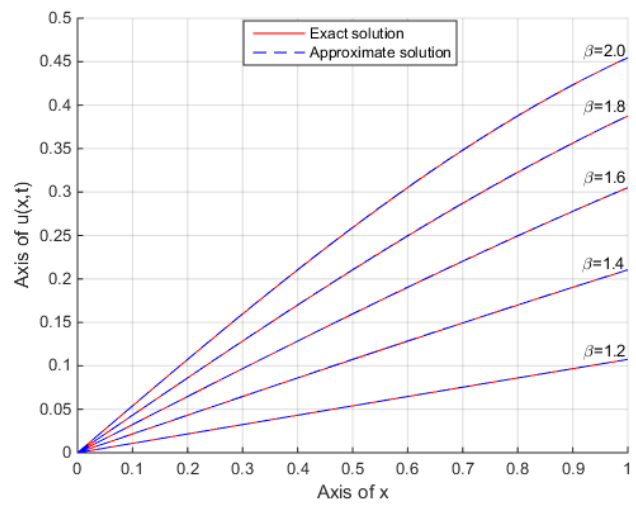

FIGURE (B). Exact and numerical solutions (Right) for $t=1$ at $\beta=1.2$ to $\beta=2$.

FiguRE 3. Comparison of numerical and exact solutions of Example 7.2 at different time and $\beta$ levels with $h=0.01, \Delta t=0.001$. 


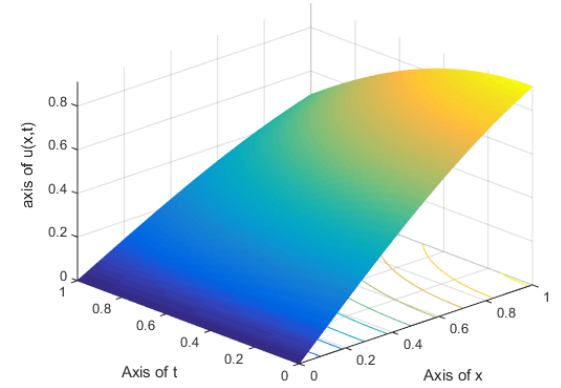

Figure (A). Numerical solution at $t=1$.

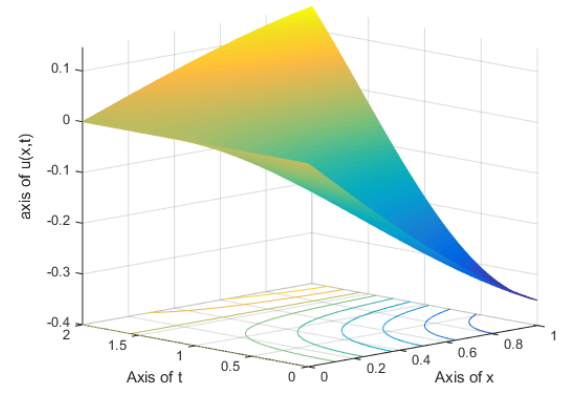

Figure (B). Numerical solution at $t=2$.

FIgURE 4. Time-space numerical solution for Example 7.2 at $t=1,2$ with $\beta=2$.

Example 7.3. In this example, we consider the telegraph Eq.(1.3) with $a=10, b=5, \omega=1$ and $\beta=1+\alpha$ in the domain $0 \leq x \leq 1$, with following initial and boundary conditions,

$$
\begin{aligned}
& u(x, 0)=\mathcal{D}_{t}^{(\alpha)} u(x, 0)=0,0<x<1, \\
& u(0, t)=0, u(1, t)=t^{1+\alpha} \sin (\pi(1+\alpha)), 0<t \leq T .
\end{aligned}
$$

and $f(x, t)=\left[\alpha(\alpha+1)+20(\alpha+1) t+25 t^{1+\alpha}+\pi^{2}(1+\alpha)^{2} x^{1-\alpha} t^{1+\alpha}\right] \sin (\pi(1+\alpha) x)$. The exact solution to this example is $u_{e}=t^{1+\alpha} \sin (\pi(1+\alpha) x)$.

$L_{2}$ and $L_{\infty}$ errors at different time step sizes $t$ for $\alpha=0.2,0.4,0.6,0.8,1.0$, with $\Delta t=0.001$ and $h=0.01$ are reported in Table 4. From Figure 5, it is clear that numerical solution coincides with the exact solution for different time and $\alpha$ levels with $\Delta t=0.001$ and $h=0.01$. The time-space graphs of numerical solution and absolute error are presented in Figure 6.

TABLE 4. $L_{2}$ and $L_{\infty}$ errors of Example 7.3 at different values of $T$ and $\alpha$ with $\Delta t=0.0001$ and $h=0.01$.

\begin{tabular}{|c|c|c|c|c|c|c|c|c|}
\cline { 2 - 9 } \multicolumn{1}{c|}{} & \multicolumn{2}{c|}{$T=0.3$} & \multicolumn{2}{c|}{$T=0.5$} & \multicolumn{2}{c|}{$T=0.7$} & \multicolumn{2}{c|}{$T=1.0$} \\
\hline$\alpha$ & $L_{2}$ & $L_{\infty}$ & $L_{2}$ & $L_{\infty}$ & $L_{2}$ & $L_{\infty}$ & $L_{2}$ & $L_{\infty}$ \\
\hline 0.2 & $5.79 E-04$ & $1.22 E-03$ & $9.57 E-04$ & $1.74 E-03$ & $1.43 E-03$ & $2.53 E-03$ & $2.20 E-03$ & $3.84 E-03$ \\
0.4 & $8.39 E-04$ & $1.59 E-03$ & $1.72 E-03$ & $3.07 E-03$ & $2.77 E-03$ & $4.87 E-03$ & $4.59 E-03$ & $8.02 E-03$ \\
0.6 & $1.01 E-03$ & $1.76 E-03$ & $2.36 E-03$ & $4.17 E-03$ & $4.10 E-03$ & $7.33 E-03$ & $7.37 E-03$ & $1.33 E-02$ \\
0.8 & $9.04 E-04$ & $1.93 E-03$ & $2.41 E-03$ & $5.20 E-03$ & $4.61 E-03$ & $9.96 E-03$ & $9.11 E-03$ & $1.97 E-02$ \\
1.0 & $7.65 E-04$ & $1.42 E-03$ & $2.44 E-03$ & $4.48 E-03$ & $5.29 E-03$ & $9.60 E-03$ & $1.20 E-02$ & $2.15 E-02$ \\
\hline
\end{tabular}




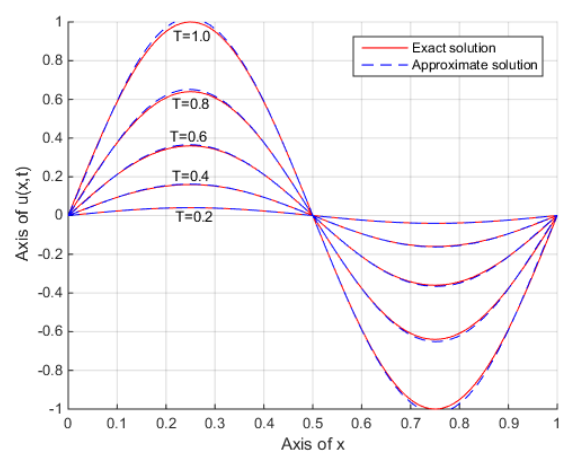

FIgURE (A). Exact and numerical solutions (Left) for $\alpha=1$ at $T=0.2$ to $T=1.0$.

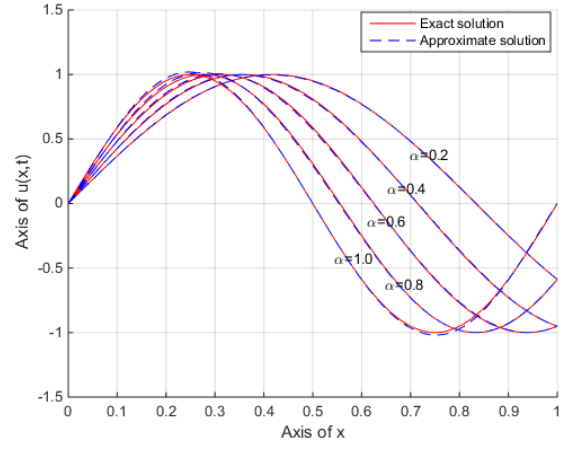

Figure (B). Exact and numerical solutions (Right) for $T=1$ at $\alpha=0.2$ to $\alpha=1$.

FIGURE 5. Comparison of numerical and exact solutions of Example 7.3 at different time and $\alpha$ levels with $h=0.01, \Delta t=0.0001$.

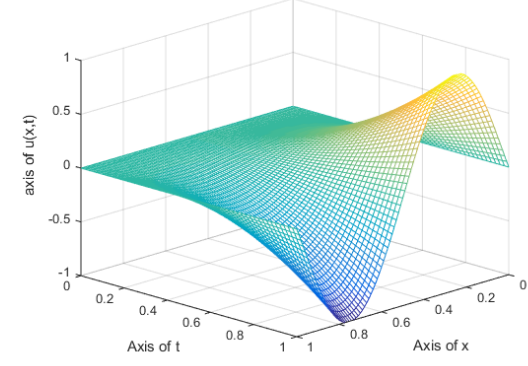

FIgURE (A). Numerical solution.

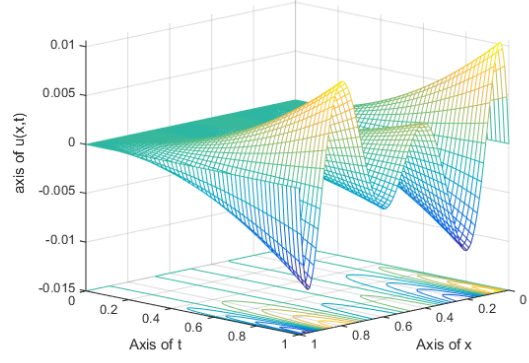

Figure (A). Absolute error.

FIGURE 6. Time-space of numerical solution and absolute error for Example 7.3 at $t=1$ and $\alpha=1$.

\section{Conclusion}

An efficient algorithm has been presented to solve the conformable time-space fractional telegraph equations. The properties of the Chebyshev polynomials of the fourth kind and conformable fractional calculus are used to reduce fractional telegraph equations into a linear system of second order differential equations and the Newmark's method is applied to solve this system. Three examples are presented to confirm the reliability and effectiveness of this algorithm. 


\section{Acknowledgments}

The authors would like to thank the referee for his/her careful reading and valuable comments, which greatly improved the paper. This research work is supported by the General Direction of Scientific Research and Technological Development (DGRSDT)-Algeria.

\section{References}

[1] Abdeljawad, T. On conformable fractional calculus. J. Comp. Appl. Math. 279 (2015), 57-66.

[2] BANAsiaK, J., AND MiKA, J. Singular perturbed telegraph equations with applications in the random walk theory. J. Appl. Math. Stoch. Anal. 11 (1998), 9-28.

[3] Berwal, N., Panchal, D., And Parihar, C. L. Haar waveleet method for numerical solution of telegraph equations. Ital. J. Pure Appl. Math. 30 (2013), 317-328.

[4] BHRAWY, A., ZAKY, M., AND MACHADO, J. Numerical solution of the two-sided space and time fractional telegraph equation via chebyshev tau approximation. J. Optim. Theory Appl. (2016).

[5] BHRAWY, A., ZAKY, M., AND MACHADO, J. Numerical solution of the two-sided spacetime fractional telegraph equation via chebyshev tau approximation. Journal of Optimization Theory and Applications 174, 1 (2017), 321341.

[6] BRAHIm, N. Numerical approach of the nonlinear reaction-advection-diffusion equation with time-space conformable fractional derivatives. AIP Conference Proceedings 2334, 060012 (2021).

[7] Brahim, N., AND Benyattou, B. Newmark method applied to the elasto-dynamic problem with slip-rate dependent friction. Journal of Concrete E Applicable Mathematics 7, 1 (2009), 70-81.

[8] Cattani, C., Srivastava, H. M., And Yang, X. J. Fractional Dynamics. de Gruyter, Berlin, 2016.

[9] Chen, J., LiU, F., AND ANH, V. Analytical solution for the time-fractional telegraph equation by the method of separating variables. Journal of Mathematical Analysis and Applications 338, 2 (2008), 1364-1377.

[10] ş. YüZBAşı. Numerical solutions of hyperbolic telegraph equation by using the bessel functions of first kind and residual correction. Applied Mathematics and Computation 287 (2016), 83-93.

[11] ş. YÜZBAŞI. A shifted legendre method for solving a population model and delay linear volterra integrodifferential equations. International Journal of Biomathematics 10, 7 (2017), p.1750091.

[12] Dehghan, M., and Ghesmati, A. Solution of the second-order one-dimensional hyperbolic telegraph equation by using the dual reciprocity boundary integral equation (drbie) method. Eng. Anal. Bound. Elements 34 (2010), 51-59.

[13] Dehghan, M., And Shokri, A. A numerical method for solving the hyperbolic telegraph equation. Numer. Methods Partial Differ. Equ. 24 (2008), 1080-1093.

[14] Heaviside, O. Electromagnetic theory. Chelsea Publishing Company, New York, Vol-2, 1899.

[15] Heydari, M., Hooshmandasl, M., and Mohammadi, F. Two-dimensional legendre wavelets for solving time-fractional telegraph equation. Advances in Applied Mathematics and Mechanics 6, 2 (2014), 247-260.

[16] JAVIDI, M. Chebyshev spectral collocation method for computing numerical solution of telegraph equation. Comput. Methods Differ. Equ. 1 (2013), 16-29.

[17] Jordan, P., ANd Puri, A. Digital signal propagation in dispersive media. J. Appl. Phys. 85 (1999), 1273-1283.

[18] Khalil, R., AlHorani, M., Yousef, A., And Sababheh, M. A new definition of fractional derivative. J. Comp. Appl. Math. 264 (2014), 65-70.

[19] Kumar, D., SingH, J., AND Kumar, S. Analytic and approximate solutions of space-time fractional telegraph equations via laplace transform. Walailak Journal of Science and Technology (WJST) 11, 8 (2013), 711-728.

[20] Kumar, S. A new analytical modelling for fractional telegraph equation via laplace transform. Applied Mathematical Modelling 38, 11 (2014), 3154-3163.

[21] Mason, J., And Handscomb, D. Chebyshev Polynomials. Chapman and Hall, CRC, New York, NY, Boca Raton, 2003.

[22] Metaxas, A., And Meredith, R. Industrial microwave heating. Peter Peregrinus, London, UK, 1993. 
[23] Mittal, R., AND Bhatia, R. Numerical solution of second order one dimensional hyperbolic telegraph equation by cubic b-spline collocation method. Appl. Math. Comput. 220 (2013), 496-506.

[24] Mohyud-Din, S. T., Yldrm, A., and Kaplan, Y. Homotopy perturbation method for one-dimensional hyperbolic equation with integral conditions. J. Phys. Sci. 65 (2010), 1077-1080.

[25] Mollahasani, N., Moghadam, M. M., and Afrooz, K. A new treatment based on hybrid functions to the solution of telegraph equations of fractional order. Applied Mathematical Modelling 40, 4 (2016), 2804-2814.

[26] Momani, S. Analytic and approximate solutions of the space- and time-fractional telegraph equations. Applied Mathematics and Computation 170, 2 (2005), 1126-1134.

[27] Muthukumar, P., AND PriYa, B. Numerical solution of fractional delay differential equation by shifted jacobi polynomials. International Journal of Computer Mathematics 94, 3 (2017), 471-492.

[28] Nazir, T., Abbas, M., and Yaseen, M. Numerical solution of second-order hyperbolic telegraph equation via new cubic trigonometric bsplines approach. Cogent Mathematics 4 (2017), 1382061.

[29] Newmark, N. M. A method of computation for structural dynamics. ASCE Journal of Engineering Mechanics Division 85 (1959), 67-94.

[30] Pekmen, B., And Tezer-Sezgin, M. Differential quadrature solution of hyperbolic telegraph equation. J. Appl. Math. (2012), 18.

[31] Podlubny, I. Fractional differential equations: an introduction to fractional derivatives, fractional differential equations, to methods of their solution and some of their applications. Academic press, 1998.

[32] Prakash, A. Analytical method for space-fractional telegraph equation by homotopy perturbation transform method. Nonlinear Engineering 5, 2 (2016), 123128.

[33] Razmjoox, N., ANd Ramezani, M. Analytical solution for optimal control by the second kind chebyshev polynomials expansion. Iranian Journal of Science and Technology, Transactions A: Science 41, 4 (2017), 10171026.

[34] SaAdatmandi, A., And Dehghan, M. Numerical solution of hyperbolic telegraph equation using the chebyshev tau method. Numer. Methods Partial Differ. Equ. 26 (2010), 239-252.

[35] SaAdatmandi, A., AND Mohabbati, M. Numerical solution of fractional telegraph equation via the tau method. Math. Rep. 17 (2015), 155-166.

[36] Samko, S., Kilbas, A., And Marichev, O. Fractional Integrals and Derivatives: Theory and Applications. Gordon and Breach, Langhorne, 1993.

[37] Sevimlican, A. An approximation to solution of space and time fractional telegraph equations by he's variational iteration method. Mathematical Problems in Engineering 2010 (2010).

[38] Sharifi, S., AND RAshidinia, J. Numerical solution of hyperbolic telegraph equation by cubic b-spline collocation method. Applied Mathematics and Computation 281 (2016), 28-38.

[39] Shivanian, E. Spectral meshless radial point interpolation (smrpi) method to two-dimensional fractional telegraph equation. Math. Methods Appl. Sci. (2015).

[40] Singh, H., Pandey, K. R., And Baleanu, D. Stable numerical approach for fractional delay differential equations. Few-Body Systems 58, 6 (2017), 156.

[41] Suleman, M., Elzaki, T., Rahman, J., and Wu, Q. A novel technique to solve space and time fractional telegraph equation. Journal of Computational and Theoretical Nanoscience 13, 3 (2016), 1536-1545.

[42] Sweilam, N., Nagy, A., ANd El-Sayed, A. Solving time-fractional order telegraph equation via sinclegendre collocation method. Mediterranean Journal of Mathematics 13, 6 (2016), 5119-5133.

[43] Weston, V., AND HE, S. Wave splitting of the telegraph equation in $\mathbb{R}$ and its application to inverse scattering. Inverse Problems 9 (1993), 789-812.

[44] YildiRIM, A. He's homotopy perturbation method for solving the space- and time-fractional telegraph equations. International Journal of Computer Mathematics 87, 13 (2010), 2998-3006.

[45] Yousefi, S. A. Legendre multiwavelet galerkin method for solving the hyperbolic telegraph equation. Numer. Methods Partial Differ. Equ. 26 (2010), 535-543. 